Revue des études hébraïques et juives

$18 \mid 2013$

Le Proche-Orient ancien à la lumière des sciences sociales

\title{
Méthodes taxinomiques comparées : Dan Sperber et Mary Douglas à propos des animaux hors catégorie
}

Comparing Taxonomic Approaches: Dan Sperber and Mary Douglas on Animals outside Taxonomy

$$
\text { חיות שאינת השוואה בטקסימוניה :גישותיהם של דן שפרבר ומרי דוגלס לגבי }
$$

\section{Anna Angelini}

\section{OpenEdition}

\section{Journals}

Édition électronique

URL : https://journals.openedition.org/yod/1834

DOI : 10.4000/yod. 1834

ISSN : 2261-0200

Éditeur

INALCO

Édition imprimée

Date de publication : 1 septembre 2013

ISBN : 978-2-85831-207-8

ISSN : 0338-9316

Référence électronique

Anna Angelini, « Méthodes taxinomiques comparées : Dan Sperber et Mary Douglas à propos des animaux hors catégorie », Yod [En ligne], 18 | 2013, mis en ligne le 08 juillet 2013, consulté le 08 juillet 2021. URL : http://journals.openedition.org/yod/1834; DOI : https://doi.org/10.4000/yod.1834

Ce document a été généré automatiquement le 8 juillet 2021.

Yod est mis à disposition selon les termes de la Licence Creative Commons Attribution - Pas d'Utilisation Commerciale 4.0 International. 


\title{
Méthodes taxinomiques comparées : Dan Sperber et Mary Douglas à propos des animaux hors catégorie
}

\author{
Comparing Taxonomic Approaches: Dan Sperber and Mary Douglas on Animals \\ outside Taxonomy \\ שיטות השוואה בטקסימוניה :גישותיהם של דן שפרבר ומרי דוגלס לגבי

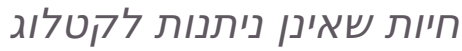

Anna Angelini

\section{Introduction}

1 Dans le cadre de cette réflexion sur l'œuvre et la pensée de Mary Douglas, ma contribution porte sur une thématique anthropologique classique et toujours pourvue d'intérêt, à savoir la taxinomie culturelle des animaux et les anomalies présentes dans chaque système taxinomique.

Dans un premier temps, je vais présenter les principes fondamentaux de la taxinomie culturelle telle qu'elle a été formulée par Mary Douglas dans son ouvrage désormais classique sur la pollution, ${ }^{1}$ mais également dans une étude postérieure, datant des années 1990, Leviticus as Literature. Elle répond dans cette dernière à plusieurs critiques en reformulant sa théorie sur la pollution dans le Pentateuque et en proposant une réflexion nouvelle sur la fonction des animaux interdits ou des aberrations taxinomiques ${ }^{2}$, aspect qui va retenir plus particulièrement mon attention. Dans un deuxième temps, je présenterai et analyserai les objections apportées par Dan Sperber³, pour conclure par une mise en relation de ces deux théories en montrant à l'aide d'un exemple comment elles peuvent interagir. 


\section{La classification taxinomique de Mary Douglas et ses aberrations}

3 Dans ses travaux sur le concept de pollution dans le domaine de la vie quotidienne et dans la sphère du sacré, ainsi que dans ses études sur le symbolisme des animaux de la tribu des Lele au Congo, Mary Douglas poursuit deux objectifs : d'une part, expliquer les raisons des règles alimentaires et des abominations du Lévitique et, d'autre part, déchiffrer le statut particulier que détiennent, chez les tribus africaines, certains animaux ${ }^{4}$. Systématiquement dans ses travaux, la réflexion de Mary Douglas porte sur les structures classificatoires, autrement dit sur le sens et la portée des taxinomies. Elle tente plus particulièrement d'analyser les éléments qui n'entrent dans aucune classification, autrement dit les aberrations taxinomiques. Elle rejette l'idée que cellesci procèdent uniquement de raisons empiriques ou pratiques. Au contraire, Douglas soutient qu'elles sont toujours l'expression d'une pensée symbolique, tant dans le domaine profane que dans le domaine sacré et religieux. Elle en déduit donc l'existence d'une relation directe entre anomalie et pensée symbolique.

Examinons à présent la manière dont Mary Douglas envisage cette relation. Selon l'anthropologue, toute classification est le produit d'un système déterminé et donc, subjective et culturelle. Comme aucun schéma classificatoire ne peut rendre compte de l'infinie diversité de l'expérience, tout système de classification va inéluctablement donner lieu à des anomalies, car certains éléments demeurent toujours réfractaires à leur inclusion dans un schéma donné. Ces anomalies ne se définissent pas par leur nature intrinsèque, mais sont le produit de la classification elle-même, classification qui varie substantiellement d'une culture à l'autre ${ }^{5}$. Il n'existe pas, d'après Mary Douglas, de système qui ne produise pas d'anomalies taxinomiques. Ces éléments qui échappent à toute classification sont ambigus, équivoques et par conséquent dangereux et de ce fait, menacent tout le système. Pour éviter le risque de sa totale remise en cause, le système se voit contraint de trouver, dans sa propre logique, des moyens pour contrôler et réduire ces anomalies. Ainsi, chaque culture élabore des solutions propres destinées à sauvegarder sa structure taxinomique. Souvent, la solution adoptée consiste à attribuer une valeur symbolique à ces éléments incarnant des aberrations taxinomiques: le système recourt ainsi à la métaphore soit pour les contrôler, les réduire, voire les éliminer, soit pour les faire intervenir, précautionneusement, dans certains rituels.

5 Arrêtons-nous à présent sur le cas particulier du Lévitique en interrogeant les lois définissant ce qu'est une " abomination » (sheqets), les règles alimentaires qui découlent de cette définition ainsi que les règles de conduite à adopter en cas de contact avec un tel élément. En refusant d'une part une interprétation exclusivement médicale, allégorique ou morale et d'autre part, le caractère arbitraire du code législatif donné par Dieu à son peuple par l'intermédiaire de Moise, Mary Douglas concentre son attention sur l'opposition entre sainteté (qedushah) et abomination (sheqets). Elle note que les préceptes du Lévitique portent en effet sur la notion de sainteté, conçue à la fois comme "séparation » et comme "intégrité, totalité, plénitude ». Le code normatif régissant la distinction entre le pur et l'impur dans le domaine alimentaire n'est qu'un exemple parmi les multiples champs d'application de cette conception générale de la sainteté comprise comme "l'unité, l'intégrité, la perfection de l'individu et de ses semblables $»^{6}$. 
Quel critère le Pentateuque retient-il pour définir la pureté des animaux ? La pureté procède de la sainteté et celle-ci découle de la séparation : il ne faut donc pas mélanger des composants de catégories différentes, les espèces de la création devant garder leur caractère distinct. Des interdictions apparemment incompréhensibles trouvent ainsi une explication : c'est le cas par exemple de Deutéronome 22.10 « Tu ne laboureras pas avec un bœuf et un âne ensemble ", sachant que par ailleurs, dans plusieurs passages de l'Exode, du Deutéronome et des Prophètes, les deux animaux sont toujours associés en qualité d'outils du travail de l'homme .

7 La pureté est donc comprise comme conformité aux règles de classement: par conséquent les animaux qui ne font pas entièrement partie de leur classe, les hybrides, ceux qui sont membres imparfaits de leur espèce, sont impurs. S'abstenir de la consommation des êtres qui ne se conforment pas aux règles de classement signifie donc, métaphoriquement, maintenir cette intégrité du corps et de l'esprit. C'est à partir de cette manière métaphorique de comprendre la notion de sainteté que procèdent toutes les règles de l'alimentation.

C'est cette même logique qui préside aux lois concernant la morale sexuelle. L'exemple $\mathrm{du}$ mulet est à ce titre très suggestif : cet animal mélange traits du cheval et de l'âne alors même que Lévitique 19.19 interdit l'accouplement de deux espèces d'animaux et le mélange de deux espèces de semences. Selon le midrach, Anah, l'homme qui a créé le mulet par l'accouplement de l'âne domestique et de l'âne sauvage, est lui-même le fils d'une relation incestueuse; il se spécialise dans la création d'hybrides et pour cette raison, il est puni par Dieu. Comme une sorte de contagion, la pollution générée par l'inceste, dont Anah est le résultat, est destinée à se perpétuer en produisant à son tour l'hybridation de deux espèces animales. De plus, une correspondance est établie entre l'hybridité biologique et la conduite sexuelle ${ }^{8}$.

9 Mary Douglas observe aussi que la structure de Lévitique 11, chapitre portant sur les interdits alimentaires, suit la structure de Genèse 1.1-2.4 qui comporte le premier récit de la création. Le langage, la classification et la division en trois groupes d'animaux terrestres, aquatiques, volatiles - sont les mêmes.

Les livres du Lévitique et de la Genèse ont également en commun le thème de l'alliance. Les animaux purs partagent avec l'homme, auquel ils sont pourtant soumis, l'alliance avec Dieu. Ils sont intégrés dans ce système où, dit Mary Douglas, le sacré et le profane ne sont pas tout à fait distincts, car appartenant tous les deux au domaine religieux. Par conséquent, «ce que celui-ci (l'homme) peut manger sans contracter d'impureté peut aussi être offert à Dieu en sacrifice $»^{9}$. Le reste demeure à l'extérieur du système et doit être évité. Le but est le même: le code alimentaire et le code du sacrifice sont une invitation faite à Israël de se joindre à l'œuvre divine de la création par une vie conforme à la manière dont Dieu créa le monde et par le respect de l'alliance.

11 À l'intérieur de ce schéma, le trait essentiel qu'il faut préserver est celui de l'intégrité. Pour réduire les anomalies et maintenir son intégrité, le système symbolique du judaïsme antique choisit de qualifier ces anomalies d'« impures » - mais il ne s'agit que d'un terme technique du culte ${ }^{10}$. Il faut par conséquent les éviter et s'abstenir de tout contact avec elles, car celui-ci entraînerait la souillure et mettrait l'individu dans un état contagieux incompatible avec le service du culte. De cette façon, la règle qui commande d'éviter les choses "anormales" affirme et renforce les définitions auxquelles ces anomalies dérogent. 


\section{La critique de Dan Sperber}

12 Dan Sperber, sur la base de ses études sur l'expression linguistique du symbolisme, parvient à mieux cerner le rapport entre anomalie et symbolisme mis en évidence par Mary Douglas. Il ajoute des éléments à sa réflexion sur la taxinomie des animaux en introduisant la notion de «norme idéale » définie comme la « conjonction de la plupart des propositions contingentes sur la faune $»^{11}$. Si tous les individus d'un genre possédaient effectivement tous les traits que leur définition taxinomique leur attribue virtuellement, alors tous ces individus seraient des animaux exemplaires, autrement dit des animaux parfaits. Cela signifie que la norme idéale ne découle pas de l'observation taxinomique du monde réel où des fragments ou des approximations de cette représentation idéale interviennent toujours comme points de référence ou de comparaison. Pour mieux comprendre ce concept, arrêtons-nous sur son exemple du $\operatorname{lion}^{12}$. Le « vrai » lion, le lion parfait, ou le modèle du lion, attaque et rugit toujours, il a une crinière majestueuse et des griffes tranchantes qui brillent, tandis que le lion «moyen », qui provient de la réalité de l'expérience, est bien différent: il n'est pas particulièrement féroce ni courageux, il arrive que sa crinière ne soit pas si majestueuse, il baille plus souvent qu'il ne rugit, etc. Ce n'est donc pas la taxinomie en elle-même, mais cette norme idéale qui engendre des anomalies, car le monde naturel s'écarte ostensiblement de la norme (et l'espèce s'écarte de la norme du genre, et le genre s'écarte de la norme du règne auquel il appartient, etc.) ; tandis que la taxinomie qui ressort de l'observation du monde naturel est, d'après Sperber, beaucoup plus « élastique » et ne produit pas d'anomalies.

13 Il existe donc une représentation sélective et normative à partir de laquelle on distingue, pour chaque espèce, d'un côté les animaux exemplaires et de l'autre, les anomalies remarquables : les représentations conceptuelles de ces deux typologies font l'objet d'un traitement symbolique, c'est-à-dire qu'elles peuvent être placées «entre guillemets $»^{13}$.

14 La critique de Dan Sperber nous est doublement utile. D'une part, elle nous permet de mieux comprendre pourquoi ce ne sont pas uniquement les animaux anormaux et les monstres qui font l'objet d'une représentation symbolique, mais aussi les animaux parfaits ou exemplaires. D'autre part, elle nous aide à affiner le schéma de Mary Douglas sur les classifications du Lévitique en ajoutant un niveau de différenciation supplémentaire. La Bible ne distingue pas seulement entre les animaux ordinaires que l'on peut manger et les animaux interdits. Lorsqu'elle donne des indications sur le sacrifice, elle distingue entre plusieurs niveaux de perfection :

Si quelqu'un offre à YHWH un sacrifice de communion pour s'acquitter d'un vœu ou pour faire un don volontaire, de gros ou de petit bétail, l'animal devra, pour être agréé, être sans défaut ; il ne s'y trouvera aucune tare. Vous n'offrirez pas à YHWH d'animal aveugle, estropié, mutilé, ulcéreux, dartreux ou purulent. Aucune partie de tels animaux ne sera déposée sur l'autel à titre de mets pour YHWH. Tu pourras faire le don volontaire d'une pièce naine ou difforme en gros ou en petit bétail, mais pour l'acquittement d'un vœu elle ne sera point agréée. (Lv. 22.21-23)

Seuls les animaux qui se rapprochent le plus possible de la norme idéale conviennent à l'accomplissement d'un vœu et peuvent faire l'objet d'un sacrifice. Je pense donc que cette nouvelle définition donnée par Sperber, au lieu de contredire le système de Mary Douglas, peut s'y insérer et enrichir ce schéma ${ }^{14}$. 


\section{Un exemple}

16 Je voudrais à présent discuter un exemple apte à montrer de quelle façon ces deux théories peuvent être conjointement mises à contribution. On prendra en considération un cas particulier d'anomalie à l'intérieur de la taxinomie des animaux bibliques, Léviathan. Bien connu au-delà du cercle restreint des biblistes, il s'agit de l'énorme monstre biblique créé par Dieu au début de l'univers. Il est décrit dans le texte biblique de plusieurs façons. Les Psaumes l'envisagent comme un monstre créé par Dieu parmi les autres tanninim, êtres géants ou grands poissons peuplant les mers, vaincu au début de la création et laissé au fond de la mer d'où il sortira à la fin des temps pour une dernière lutte avec Dieu ${ }^{15}$. Par contre, le livre de Job nous donne une description beaucoup plus précise où l'animal est présenté comme une sorte d'énorme crocodile avec des écailles impénétrables de pierre, des flammes qui jaillissent de sa gorge, des naseaux qui crachent de la fumée, capable d'écraser tous les autres êtres vivants (Jb. 40.15-41.26, la plus longue description biblique de ce monstre). La première question qui se pose est donc: quelle est sa place dans la taxinomie? S'agit-il d'un poisson, d'un crocodile ou plutôt d'un dragon? Le livre de Job associe Léviathan à une autre figure énigmatique, Béhémoth, être de taille démesurée habitant les marécages (Jb. 40.15-24). Défini comme la première créature de Dieu, il est le roi des bêtes sauvages, capable de boire d'un seul coup toute l'eau du Jourdain. Il est clair que toute tentative d'identifier la place de ces deux créatures dans le cadre de la taxinomie des animaux est vouée à l'échec, car ce sont des monstres mythologiques liés aux récits de la création et au mythe cosmogonique ${ }^{16}$. Le rôle qu'ils jouent dans la tradition biblique a des parallèles, très précis et nombreux, dans toutes les mythologies proche-orientales ${ }^{17}$. Ils se situent hors taxinomie, fait qui explique leur traitement symbolique: ils représentent les ennemis de Dieu et de son ordre.

17 Ce récit biblique du monstre subit, dans la tradition juive post-biblique, des transformations importantes. Léviathan n'est plus simplement un dragon ayant mené son combat au début de la création. Dans la littérature intertestamentaire et dans les sources rabbiniques il est destiné au banquet des justes à la fin des temps. Ces textes nous décrivent un grand repas préparé par Dieu lors de l'avènement de l'ère messianique, réservé à tous ceux qui se sont bien comportés. À cette occasion les Juifs pourront finalement manger tout ce qu'il leur a été interdit tout au long des siècles : ils jouiront d'une juste compensation et Léviathan et Béhémoth feront l'objet de ce banquet. Cette idée se retrouve par exemple dans l'Apocalypse syriaque de Baruch 29.4 :

Et Béhémoth apparaîtra hors de son lieu propre, et Léviathan remontera (du fond) de la mer tous deux, monstres gigantesques que j'ai réservés en vue de ce temps, pour servir de nourriture à tous ceux qui survivront ${ }^{18}$.

Cette croyance en cours dans le monde juif dénote une nouvelle manière de conceptualiser les monstres en question. Dans l'iconographie médiévale, Léviathan a perdu ses traits de dragon, très marqués dans le récit biblique, pour devenir tout simplement un grand poisson. C'est ainsi qu'il figure dans un manuscrit de la bibliothèque Ambrosienne (illustration 1) : la partie supérieure représente Léviathan et la partie inférieure, la table messianique des justes. 
Illustration 1 : MS Ambrosienne B32 Inf., fol. 136r

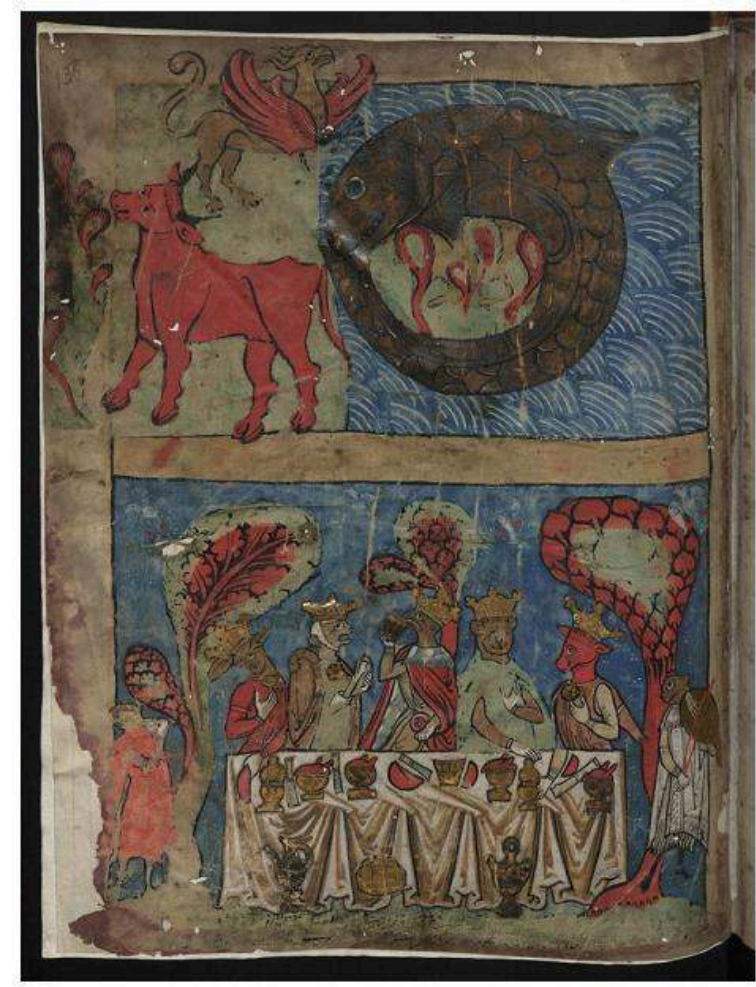

19 Dans le même esprit, les récits talmudiques mentionnent l'animal comme une grande bête de mer, le roi des poissons, soumis par Dieu à un traitement particulier :

[...] Le Saint Béni Soit-Il créa également Léviathan, mâle et femelle. [...] Il châtra le mâle, tua la femelle et la conserva dans le sel pour le repas des justes dans le monde à venir ${ }^{19}$.

20 Léviathan devient donc un poisson conservé dans la saumure. Dans ce nouveau contexte, Léviathan, au lieu d'être un hybride monstrueux, est replacé à l'intérieur de la taxinomie ou mieux, pour adopter le concept proposé par Sperber, il est devenu l'animal parfait, le représentant de la norme idéale du genre des poissons. Béhémoth, quant à lui, devient le représentant de la catégorie des quadrupèdes, c'est-à-dire un bœuf géant (illustration 2) ${ }^{20}$, représenté à côté de Léviathan dans un manuscrit de la British Library (illustration 3) ${ }^{21}$. 
Illustration 2 : MS British Library Add. 11, 639, fol. 519a

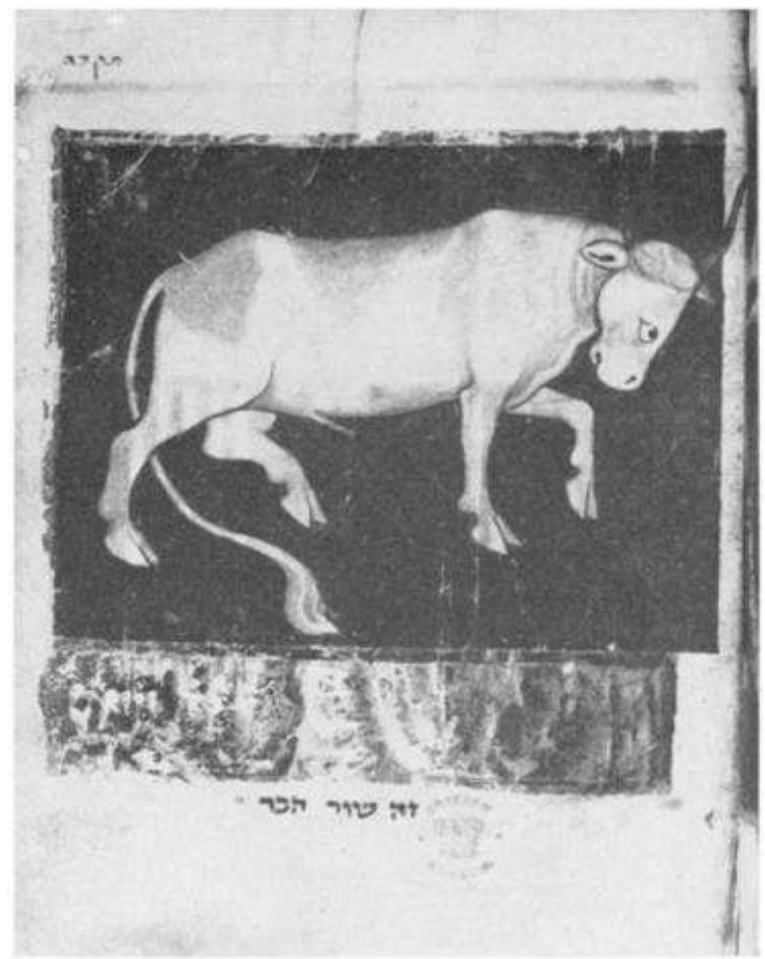

Illustration 3 : MS British Library,

Add. 11, 639, fol. 518b

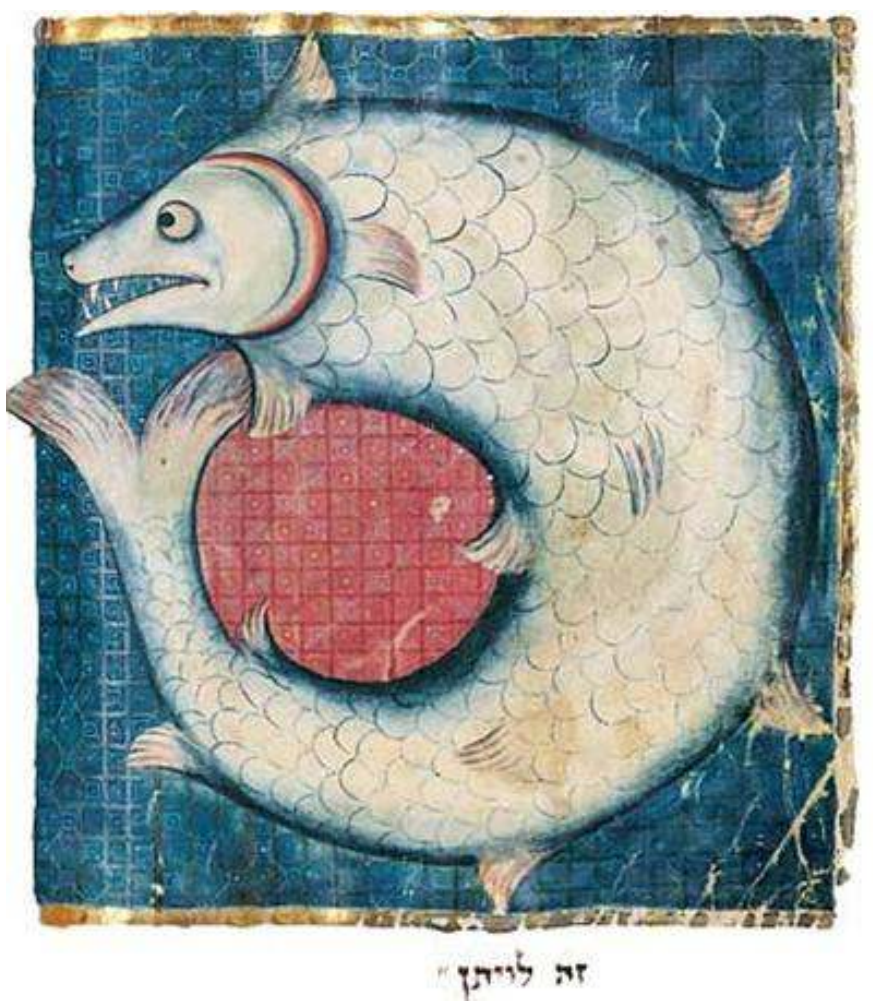



vue de ce banquet final ${ }^{22}$. Les débats rabbiniques sur la pureté des animaux deviennent vifs. Le traité Hullin, qui cite le texte de Job, affirme que Léviathan est un poisson pur, en considérant les «boucliers " comme les écailles qui le recouvrent et «les tessons aigus sous lui » comme les nageoires avec lesquelles il se meut ${ }^{23}$. Selon Lévitique Rabbah 13.3, Léviathan et Béhémoth mourront en engageant une lutte cruelle l'un contre l'autre et les rabbins se demandent alors s'il s'agit d'une méthode d'abattage kasher. Comment expliquer l'insistance des rabbins sur ces détails? Ceci se comprend justement à la lumière des théories de Mary Douglas qui portent sur la nécessité, pour les Juifs, de maintenir la pureté comme garantie, à travers les siècles, de l'identité juive, comme marque de séparation entre l'homme et l'animal d'un côté, entre Israël et les gentils de l'autre. Pour bien sceller cette séparation, il faut trouver des représentants symboliques du règne animal qui soient au plus près de la perfection et conviennent au repas qui sera « Le Repas » par excellence.

En poursuivant jusqu'au bout cette logique tripartite énoncée dans Genèse et Lévitique, les textes et l'iconographie du Moyen Âge produisent une troisième figure, représentant à la fois le règne animal des volatiles et la nourriture permise dans cette catégorie d'animaux (illustration 4).

\section{Illustration 4 : MS British Library,}

Add. 11, 639, fol. 517b

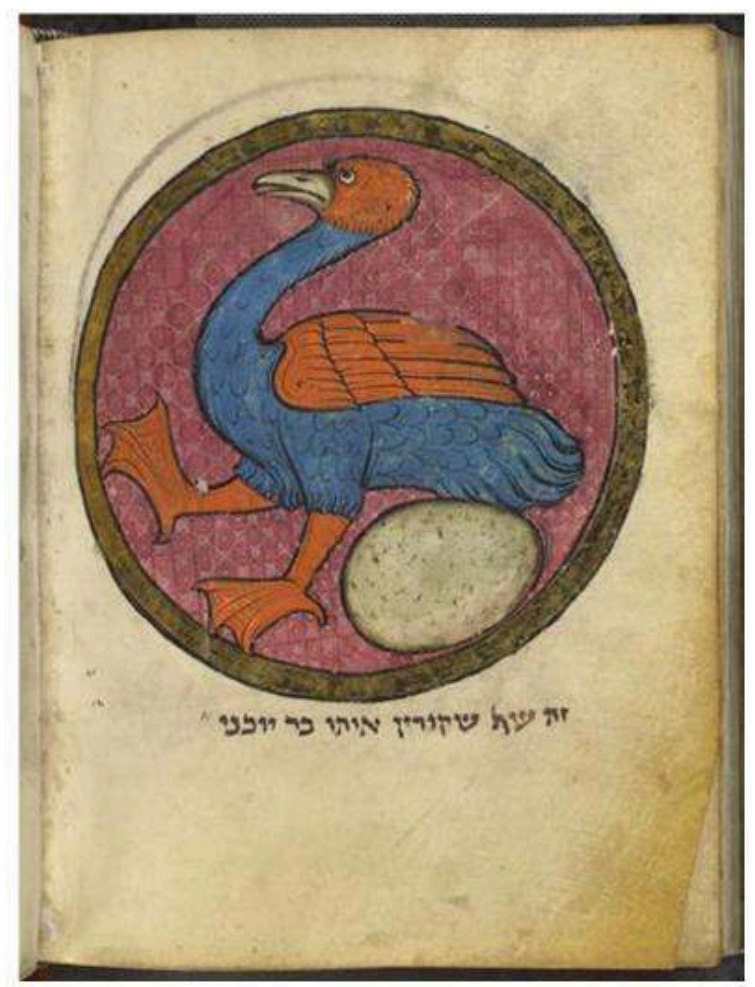

Il s'agit de Ziz, l'oiseau céleste, créé par l'exégèse médiévale et figurant parmi les plats du festin des justes. Il n'est présent ni dans la Bible, ni dans la littérature intertestamentaire mais souvent mentionné dans les sources rabbiniques ${ }^{24}$. Son nom est mis en rapport avec sa destination alimentaire par l'étymologie créative des 
rabbins : «Pourquoi a-t-il été appelé Ziz ? Parce qu'il possède différents goûts : le goût de ceci (zeh) et le goût de cela (zeh)» (Lv. R 22.10).

Ces trois animaux sont réunis dans la miniature de la bibliothèque Ambrosienne, surplombant la table du banquet (illustration 1) et Lévitique Rabbah 22.10 nous éclaire à ce propos :

Comme compensation pour l'interdiction de certains poissons, je t'ai permis Léviathan, un poisson pur; comme compensation pour l'interdiction de certains oiseaux, je t'ai permis $\mathrm{Ziz}$, un oiseau pur [...]; comme compensation pour l'interdiction de certaines bêtes, je t’ai permis Béhémoth [...].

\section{NOTES}

1. M. Douglas, Purity and Danger. An Analysis of Concepts of Pollution and Taboo, New York, Routledge, 1966 (nous citons ici l'édition française : De la souillure. Essai sur les notions de pollution et tabou, trad. Anne Guérin, Paris, Maspero, 1971).

2. M. Douglas, Leviticus as Literature, Oxford, Oxford U.P., 1999 (nous citons ici l'édition française : L'anthropologue et la Bible. Lecture du Lévitique, trad. Jean L'Hour, Paris, Bayard, 2004). Parmi les approches critiques de la théorie de Mary Douglas on retient ici l'article de Tambiah (S. J. Tambiah, "Animals are Good to Think and Good to Prohibit" Ethnology 7, 1969, pp. 423-459) et la révision de cette théorie par Jacob Milgrom dans son commentaire du Lévitique (Jacob Milgrom, Leviticus 1-16, The Anchor Bible, New York, Doubleday, 1991, pp. 718-736).

3. D. Sperber, « Pourquoi les animaux parfaits, les hybrides et les monstres sont-ils bons à penser symboliquement?», L'Homme 15(2), 1975, p. 5-34.

4. Douglas elle-même affirme qu'elle n'aurait jamais été amenée à une lecture anthropologique du Lévitique sans son travail de terrain en Afrique dont on peut lire les résultats dans de nombreuses études consacrées à ce sujet. Pour le rôle joué par les animaux dans le symbolisme religieux des Lele au Congo et les similarités mises en évidence avec les classifications du Lévitique, voir par exemple Implicit Meanings. Essays in Anthropology, Londres, Routledge, 1975, pp. 47-62.

5. Voir aussi sur ce sujet M. Douglas, "The Pangolin Revisited: a New Approach to Animal Symbolism" in R. Willis (éd.), Signifying Animals. Human Meaning in the Natural World, Londres-New York, Routledge, 1990, pp. 25-36.

6. M. Douglas, De la souillure, op. cit. p. 73.

7. Ex. 23.4, 21.33, 23.12 ; Dt. 5.14, 28.31 ; Ex. 20.17 ; Jb. 6.5. L'âne et le bœuf, cités presque toujours ensemble, sont considérés comme faisant partie de la famille et ont droit au repos du shabbat.

8. Gn. 36.24 : « Anah qui a trouvé les yemim dans le désert ». À partir de la moitié du IV siècle, les rabbins commencent à discuter le sens du terme ha-yemim. Dans son commentaire sur ce verset, Jerôme cite différentes définitions du mot, tirées des sources juives : «mer » en lisant yammim ; "sources chaudes" en lisant hammim; une espèce d'âne qui court très vite appelée yemin, obtenue par Anah par le croisement de l'âne domestique et l'âne sauvage; enfin « mulets ». Cette dernière interprétation était, selon Jerôme, la plus répandue parmi les Juifs : on croyait que Anah était le premier à avoir élevé le mulet, en introduisant une nouvelle espèce animale contraire aux lois naturelles. Les sources rabbiniques connaissent très bien cette quatrième explication et donnent des détails à ce sujet, en disant que Anah était lui-même un bâtard, sa mère étant aussi 
la mère de son père. Pour punir cet accouplement contre nature, Dieu amena sur la terre le serpent d'eau à la morsure mortelle, résultat du croisement entre une vipère et un lézard. Voir Genèse Rabbah 82.15 ; Yerushalmi Berakhot I, 12b ; Pesahim 54a ; L. Ginzberg, Encyclopaedia Judaica, CD-rom edition, Jérusalem, Keter Publishing House, 1997, s.v. Anah.

9. M. Douglas, L'anthropologue et la Bible, op. cit. p. 166.

10. Sur le concept de sheqets et sa traduction, voir aussi M. Douglas, "Sacred Contagion”, in J. F. A. Swyer (éd), Reading Leviticus. A conversation with Mary Douglas, JSOT Suppl 227, Sheffield, Sheffield Academic Press, 1996, pp. 86-106.

11. D. Sperber, « Pourquoi les animaux... », art. cité, p. 25.

12. Ibidem p. 24.

13. Ibid., p. 16.

14. Dans Leviticus as Literature, Douglas, en accueillant cette critique, introduit une nouvelle distinction : les animaux sont classés non seulement selon la sphère à laquelle ils appartiennent, mais aussi selon leur degré de sainteté. Ainsi, parmi les animaux purs, autorisés à la consommation, certaines espèces sont sélectionnées pour l'autel. Voir M. Douglas, L'anthropologue et la Bible, op. cit. p. 161-178.

$15 . \quad$ Ps. 74.13-14:

« Tu as maîtrisé la mer par ta force, fracassant la tête des dragons sur les eaux ; tu as écrasé les têtes de

Léviathan, le donnant à manger à une bande de chacals. »Ps.104.25-26 : «

Voici la mer, grande et vaste de tous côtés, où remuent, innombrables, des animaux petits et grands. Là, vont et viennent les bateaux et Léviathan que tu as formés pour jouer avec lui. » Voir aussi

: «Ce jour-là, le Seigneur interviendra avec son épée acérée, énorme, puissante contre Léviathan,

27.1 le serpent fuyant, contre Léviathan, le serpent tortueux, il tuera le Dragon de la mer. »

16. Une telle identification fut tentée depuis l'Antiquité jusqu'à nos jours: voir à ce sujet A. Caquot, «Le Léviathan de Job 40.25-41.26», Revue biblique 99 (1), 1992, p. 40-69 ; A. Angelini, «Percorsi di zoologia fantastica nell'Antico Testamento», in C. Rosenzweig, A. Callow, V. Brugnatelli, F. Aspesi (éds), Florilegio filologico linguistico. Haninura de Bon Siman a Maria Luisa Mayer Modena, Milan, Cisalpino, 2008, p. 1-16.

17. Voir, entre autres, avec leurs bibliographies respectives, E. Lipiński, s.v. Liwjātān in G.J. Botterweck, H. Ringgren (éds.), Theologisches Wörterbuch zum Alten Testament, vol. 4, Stuttgart, Kohlhammer, 1982-1984 ; C. Uehlinger, s.v. Leviathan in K. van der Toorn, B. Becking, P.W. van der Horst (éds.), Dictionary of Deities and Demons in the Bible, Leiden-Boston-Köln, Brill, $2^{\mathrm{e}}$ éd. 1999.

18. Apocalypse de Baruch, trad. et com. Pierre Bogaert, 2 vol., Paris, Cerf, SC, 1969. Voir aussi 1Enoch 60.7-9 ; 4Esdras 7.47-52; Lv R 13.3 ; 22.10; Nb R 21.18; Pirqé deRabbi Eliézer 10. Pour une analyse complète de ces sources voir K. W. Whitney, Two Strange Beasts: Leviathan and Behemoth in Second Temple and Early Rabbinic Judaism, Winona Lake (Indiana), Eisenbrauns, 2006.

19. Baba Bathra $74 \mathrm{~b}$ (ma traduction). On trouve dans ce passage la même affirmation concernant Béhémoth.

20. Au Moyen Âge Béhémoth est aussi appelé Shor ha-bar: voir le petit sous-titre de cette miniature et les témoignages talmudiques.

21. Pour l'analyse iconographique de ces miniatures, voir J. Gutmann, "When the Kingdom Comes, Messianic Themes in Medieval Jewish Art”, Art Journal 27 (2), 1967-1968, pp. 168-175.

22. Description détaillée dans 4Esdras 7.47-52.

23. Hullin $67 \mathrm{~b}$ à propos de $\mathrm{Jb}$.

«Quel orgueil ! de si solides boucliers! bien clos, scellés, pressés ! et deJb.

41. 22 : « Il a sous lui des tessons aigus, comme une herse, il se traîne sur la vase ».Pour les écailles et les nageoires dans le Pentateuque, voir Lv. 11.9-11 et Dt. 14.9-10. 
24. Baba Bathra 73b; Gn. R 19.4; Midrash Konen 26. D'autres sources sont indiquées par L. Ginzberg, The Legends of the Jews, 7 vol., Philadelphie, Jewish Publication Society of America, 1967-1969, vol. 1, p. 26ss, vol. 5, pp. 113ss.

\section{RÉSUMÉS}

Cet article aborde un sujet anthropologique classique et toujours pourvu d'intérêt, à savoir la taxinomie culturelle des animaux et les anomalies présentes dans chaque système taxinomique. Il compare la théorie de Mary Douglas à celle de Dan Sperber et montre, à travers un exemple, comment les deux approches peuvent fonctionner de pair.

This article proposes a reflection on a classic topic in cultural anthropology: the cultural taxonomy of animals and the anomalies existent in every such taxonomic system. In the first part, I compare the theories of Mary Douglas and Dan Sperber while in the second part, I show, using an example, how those two approaches can work together.

\section{INDEX}

\section{מילות מפתח}

המזרח התיכון, ספר ויקרא, הים התיכון, טקסימוניה תרבותית, חיה מושלמת, אנומליה:

טקסימונית

Mots-clés : Lévitique, taxinomie culturelle, animal parfait, anomalie taxinomique, Sperber Dan (1942-), Douglas Mary (1921-2007)

Index géographique : Méditerranée, Proche-Orient

Keywords : Leviticus, cultural taxonomy, perfect animal, taxonomic anomaly, Sperber Dan (1942-), Douglas Mary (1921-2007), Mediterranean, Middle East

\section{AUTEUR}

\section{ANNA ANGELINI}

Université de Genève 